\title{
Le développement durable en pratique : l'exemple des Éco-quartiers à Montréal
}

\author{
Geneviève Cloutier et Pierre Hamel ${ }^{1}$ \\ Université de Montréal
}

\section{Introduction}

Le thème du développement durable fait maintenant partie de la culture sociale et politique des pays occidentaux. À la suite de l'action des mouvements verts et d'une prise de conscience collective à l'égard de l'importance des enjeux environnementaux en ce qui a trait à l'avenir des sociétés contemporaines, un consensus s'est dégagé aussi bien à l'échelle locale, régionale que nationale. Il est nécessaire de passer à l'action d'une manière diversifiée pour atteindre à long terme les objectifs inhérents au programme d'un développement durable. C'est dire qu'une majorité d'acteurs sociaux et politiques sont d'accord pour intervenir d'une manière ponctuelle sur une multitude d'objets et de choses que nous associons à la nature en modifiant leurs attitudes et leurs pratiques passées - avec un souci de mieux respecter les équilibres écologiques ou en essayant de les restaurer - en l'absence d'un modèle éprouvé qui garantirait à moyen terme l'atteinte des objectifs globaux et ultimes du développement durable. En d'autres mots, même s'il est difficile d'entrevoir pour le moment comment il sera possible à long terme d'assurer à la fois une plus grande justice sociale et la protection de l'environnement pour l'ensemble de la planète, il est néanmoins nécessaire de passer à l'action et de modifier nos habitudes de vie en commençant par ce qui est plus près de nous.

C'est le cas des résidus domestiques qui constituent une part importante des déchets que produisent les sociétés industrielles avancées reposant sur la consommation de masse. L'enjeu de la gestion de ces résidus se situe autant du côté des ressources non renouvelables mises à contribution dans la production industrielle que du côté des effets négatifs des déchets déversés dans l'environnement et qui résultent à la fois de la production et de la consommation. La réduction, la réutilisation et le recyclage peuvent alors être combinés et fournir les bases d'un modèle d'intervention qui engage la responsabilité des entreprises, des ménages et des gestionnaires publics.

À Montréal, même si la collecte sélective qui permet de mettre en œuvre le recyclage est passée dans les mœurs, celle-ci demeure insuffisante par rapport aux objectifs que veut atteindre le gouvernement du Québec. De plus, une hausse anticipée des coûts de la collecte sélective à l'été 2002 a soulevé plusieurs questions concernant l'efficacité et la pertinence du système de recyclage en vigueur. Est-ce qu'il ne faudrait pas repenser de fond en comble les pratiques actuelles de gestion ? Quel rôle jouent les Éco-quartiers sur ce plan ? Qu'est-ce qui peut permettre d'améliorer le recyclage des résidus domestiques sur le territoire de Montréal, mais aussi dans les autres villes du Québec?

Afin de répondre à ces questions, nous avons analysé l'an dernier le fonctionnement des Éco-quartiers à Montréal à partir d'une double approche qualitative et quantitative ${ }^{2}$. C'est une partie des résultats provenant de cette étude que nous présentons dans les pages qui suivent. Ainsi, nous commençons par situer les Éco-quartiers et leur mandat dans leur contexte. Ensuite, nous considérons les principaux obstacles qui limitent encore le recyclage de nos jours. Cela nous conduit à examiner une approche 
différente qui est celle mise en place par la ville de Guelph en Ontario. Enfin, nous considérons les caractéristiques sociodémographiques des quartiers urbains montréalais afin de mieux cerner les défis qu'ils représentent pour des acteurs communautaires comme les Éco-quartiers.

À la lumière des changements de mentalité qui sont survenus ces dernières années en matière d'environnement et de recyclage, et étant donné la responsabilité accrue des municipalités à cet égard, il nous est apparu utile de rendre compte des initiatives qui ont été mises de l'avant à Montréal grâce aux Écoquartiers. Ces initiatives engagent l'administration municipale, mais aussi les citoyens et les organismes communautaires qui interviennent sur les questions environnementales. Il s'agit là d'une forme de partenariat dont nous mesurons encore mal les incidences, et ce, tant pour les administrations municipales que pour les autres catégories d'acteurs engagés dans ces projets.

\section{Une structure décentralisée de soutien technique à la collecte sélective}

L'efficacité des systèmes de recyclage des résidus domestiques des pays industrialisés dépend actuellement de la participation des ménages aux activités du tri. En effet, les administrations municipales comptent essentiellement sur la volonté et sur la disposition des ménages à participer au programme afin d'accroître leur performance sur ce plan. Toutefois, nous savons que cette participation est conditionnée par certains facteurs. La diffusion de l'information sur les façons de faire et les raisons de recycler sont notamment primordiales pour encourager l'adhésion de la population au système de collecte sélective et pour rendre le système opérationnel et efficace ${ }^{3}$.

Afin de mettre en place les conditions favorables à l'adhésion de la population à la collecte sélective, la Ville de Montréal a mis sur pied, au milieu des années 1990, un réseau d'intervenants locaux ayant pour mission d'assister les ménages dans leurs tâches de collecte sélective. Divers organismes de quartier (des groupes communautaires, des YMCA, une association étudiante, etc.) se sont vu accorder un mandat d'Éco-quartier élaboré par l'administration munici- pale pour travailler à sensibiliser les résidents des quartiers de l'ancienne ville de Montréal à l'amélioration de leur cadre de vie.

\section{L'efficacité des systèmes de recyclage des résidus domestiques des pays industrialisés dépend actuellement de la participation des ménages aux activités du tri.}

Le réseau des Éco-quartiers qui fut alors mis sur pied ne se consacrait pas exclusivement à l'amélioration des $3 \mathrm{R}$ (réduction, réutilisation, recyclage) de la gestion intégrée des matières résiduelles. Les organismes voyaient également à l'amélioration de la propreté, à l'embellissement et à l'amélioration du patrimoine végétal des quartiers. Mais l'objectif prioritaire de la programmation Éco-quartiers était, au moment de sa mise en place, de poursuivre l'implantation du système de collecte sélective à domicile, entamée en 1989 à Montréal, et ce volet est, encore aujourd'hui, celui qui fait l'objet du plus grand nombre d'interventions auprès de la population. Le personnel des Éco-quartiers fournit aux ménages le matériel de soutien conçu par l'administration municipale pour la collecte sélective. Il explique également à la population, à l'aide de brochures, d'activités spéciales et de tournées dans les écoles, par exemple, comment et pourquoi le tri effectué au début de la chaîne de vie des résidus est de première importance pour la suite du processus de récupération.

En appelant à la contribution des intervenants locaux pour la sensibilisation et l'éducation de la population, l'administration montréalaise a favorisé l'implantation d'une gestion décentralisée des matières résiduelles. La structure décentralisée des Éco-quartiers contribue d'ailleurs à faciliter l'adhésion de la population aux activités de recyclage. Le travail des 51 Éco-quartiers ${ }^{4}$ de l'ancienne ville aura permis d'étendre le programme de récupération des matières recyclables en bordure de rue, qui est passé d'un taux de desserte de $24 \%$ en 1994 à une desserte presque générale en $2002^{5}$. De plus, alors que le taux de matières récupérées s'établissait à $10 \%$ à Québec, où il n'existe pas d'équivalent aux Éco-quartiers, il atteignait $18 \%$ à Montréal en 2000. 


\section{Des obstacles à l'augmentation de la récupération}

Cependant, bien que les ménages qui participent aux activités de recyclage soient aujourd'hui plus nombreux, et malgré le fait que le taux de récupération atteint par l'ancienne ville de Montréal soit plus élevé en comparaison à celui des autres grandes villes québécoises, il semble que le programme montréalais de collecte sélective ne suffise pas à l'atteinte de l'objectif de récupération que s'est donnée l'administration montréalaise. Cette dernière souhaitait atteindre un taux de récupération de $40 \%$ en 2000. À cette date, moins de la moitié de l'objectif avait été atteint. Plusieurs facteurs ont contribué et contribuent encore à freiner la progression du taux de récupération à Montréal, et même partout au Québec, cela malgré le fait que le taux de participation des ménages au recyclage a augmenté en 2002 par rapport à 2001 dans le cas de l'ancienne ville de Montréal. Dans l'ensemble des quartiers, "près de 62000 tonnes de papier, de verre, de métal et de plastique recyclables ont été recueillies en 2002, contre 57800 tonnes en 2001, une hausse de $7,3 \% »^{6}$.

\section{En appelant à la contribution des intervenants locaux pour la sensibilisation et l'éducation de la population, l'administration montréalaise a favorisé l'implantation d'une gestion décentralisée des matières résiduelles.}

Comme il a été mentionné précédemment, l'information diffusée aux ménages concernant les façons de faire le tri et ce que ce tri permet de réaliser conditionne leur participation à la collecte sélective. Dans ce contexte, il importe de faciliter l'action des ménages pour encourager leur adhésion au recyclage. Un espace domiciliaire restreint, l'accès compliqué aux infrastructures de collecte sélective, la nécessité de posséder un bac de recyclage sont, par exemple, des contraintes physiques propres aux milieux urbains denses qui peuvent freiner l'augmentation des matières récupérées. Plus généralement, la disparité des équipements de recyclage entre les régions complique la tâche des résidents. Ces der- niers doivent en effet se demander si tel plastique, bien que marqué du cercle de Moebius (symbole identifiant les matériaux recyclables), peut être recyclé dans leur région. Les cartons de lait sont recyclables à Montréal, par exemple, mais ils ne le sont pas à Québec ni à Sherbrooke. Cette situation complexifie le tri et oblige les municipalités à faire un rappel constant des consignes. Le manque de débouchés pour certains produits explique à la fois la disparité des équipements entre les régions et la stagnation du taux de récupération.

\section{La difficile augmentation du taux de récupération et les récentes hausses anticipées des coûts de la collecte invitent à s'interroger sur la pertinence de maintenir le système de collecte sélective tel qu'on le connaît actuellement.}

Aussi, les frais de la collecte étaient jusqu'à récemment presque entièrement assumés par les municipalités et leurs contribuables qui accomplissent déjà une grande partie du service de récupération en triant les résidus. Les entreprises à l'origine de la mise en marché des matières n'étaient pas tenues de payer pour leur récupération. Cette situation inéquitable décourageait certains ménages. Néanmoins, depuis l'adoption par l'Assemblée nationale du Québec du projet de loi 102, en décembre 2002, les entreprises qui produisent des matières recyclables sont tenues d'assumer une partie des coûts de la collecte sélective.

Les programmes municipaux de recyclage peuvent par ailleurs se révéler inadéquats lorsqu'ils ne tiennent pas compte des caractéristiques de la population à laquelle ils s'adressent. En effet, plusieurs programmes négligent de s'adapter au contexte social, alors même que certains facteurs sociodémographiques, tels que la connaissance du français ou de l'anglais ainsi que la capacité de lire la documentation écrite dans l'une de ces deux langues, ont une influence sur la participation des ménages à la collecte sélective ${ }^{7}$.

La difficile augmentation du taux de récupération partout au Québec et les récentes hausses anticipées 
des coûts de la collecte à Montréal invitent à s'interroger sur la pertinence de maintenir le système de collecte sélective tel qu'on le connaît actuellement.

\section{Privilégier la facilité ?}

À l'été 2002, les arrondissements de la nouvelle ville de Montréal, qui sont chargés d'octroyer les contrats pour la collecte des matières résiduelles, apprenaient que les coûts de ces contrats allaient augmenter considérablement. Les entrepreneurs en charge de la collecte des matières résiduelles à Montréal expliquent cette hausse des coûts par l'augmentation de la desserte et, par conséquent, du volume de matières à collecter (l'augmentation du volume de matières était évaluée à plus de $40 \%$ dans certains arrondissements) et par l'augmentation du prix du diesel, des assurances et des salaires des employés, depuis les derniers contrats signés en $1998^{8}$.

La hausse annoncée des coûts de la collecte, qui devaient doubler dans plusieurs arrondissements, a soulevé la question de la responsabilité de la facture du programme montréalais de collecte sélective ainsi que celle de l'efficacité de ce programme. Est-ce à la municipalité et à ses contribuables à payer les coûts de la collecte sélective ? Y aurait-il des moyens plus efficaces de récupérer les matières et de limiter la pollution? Les arrondissements avaient jusqu'au mois d'avril 2003 pour établir de nouveaux contrats de collecte sélective, et cette date butoir a stimulé la recherche de nouvelles façons de procéder dans la plupart de ces arrondissements

Certaines villes nord-américaines et européennes ont récemment mis au point des systèmes faisant le pari de faciliter l'action des ménages pour assurer un meilleur tri et, ainsi, réduire les quantités de résidus à éliminer tout en diminuant les coûts de la gestion des matières résiduelles. La ville de New York a notamment décidé de cesser, depuis l'été 2002, la collecte sélective du plastique en raison du faible nombre d'acheteurs pour cette matière sur le marché actuel. L'administration new-yorkaise a ainsi jugé qu'il était plus rentable de mettre de côté la récupération du plastique et de privilégier l'incinération de cette matière jusqu'à ce que le prix de la ressource augmente.
Pour sa part, la ville de Guelph en Ontario, qui compte environ 50000 habitants, a adopté en 1995 un système de tri simplifié des matières résiduelles, unique en Amérique du Nord, qui gagne chaque année en popularité. Le programme de Guelph, appelé « sec-humide», requiert des ménages qu'ils séparent leurs résidus liquides (les matières putrescibles) de leurs résidus solides (les ordures ménagères et les matières recyclables). Ce système n'exige ainsi qu'un tri minimal des matières. La sélection des déchets qui peuvent être récupérés se fait par la suite dans un centre de tri, de façon mécanisée.

\section{Certaines villes nord-américaines et européennes ont récemment mis au point des systèmes faisant le pari de faciliter l'action des ménages pour assurer un meilleur tri.}

Cette méthode, par le geste simplifié qu'elle requiert, assure une participation accrue des ménages sur le territoire de Guelph. Ce faisant, elle permet d'y traiter quatre fois plus de résidus que le système de collecte sélective avec bac de recyclage ${ }^{9}$. Ce système nécessite toutefois d'importantes dépenses en infrastructures pour assurer le tri mécanisé des matières et il met de côté l'objectif de responsabilisation des ménages inhérent à la collecte sélective.

\section{Le lent changement des habitudes}

Le système "sec-humide » tel qu'il est appliqué à Guelph pourrait sans doute permettre d'augmenter le volume de matières collectées à Montréal ou ailleurs au Québec. Cependant, il évacuerait complètement l'idée de responsabilisation des consommateurs inhérente à la collecte sélective de même que la notion d'éco-civisme dont les Éco-quartiers font la promotion à Montréal. Le geste du tri des matières résiduelles constitue, d'une certaine façon, une tâche supplémentaire pour les ménages, mais il favorise une prise de conscience et une mobilisation en ce qui a trait à la consommation des ressources.

En effet, bien que le processus de changement des habitudes préconisé par des organismes comme les Éco-quartiers soit plutôt lent et qu'il exige d'impor- 
tantes dépenses permettant d'assurer un soutien technique aux ménages, il s'inscrit dans une démarche de développement durable. Le principe du programme, qui prévoit qu'un organisme local bien implanté dans le quartier assume la transmission des informations et la sensibilisation constante à l'importance de recycler et de bien trier les matières, poursuit l'objectif d'instaurer une nouvelle culture de responsabilisation des résidents envers leur environnement. D'ailleurs, la structure décentralisée du programme Éco-quartier favorise, dans les faits, une meilleure connaissance des caractéristiques de la population de chaque quartier et facilite la mobilisation de cette population. Les observations des Éco-quartiers ont notamment permis à l'administration municipale de modifier son matériel de soutien de façon à mieux rejoindre les groupes de population ne lisant pas le français (en utilisant des pictogrammes pour donner les consignes du tri) et de faciliter la tâche à ceux qui sont limités dans leur mobilité (en rendant les bacs de recyclage plus faciles à transporter).

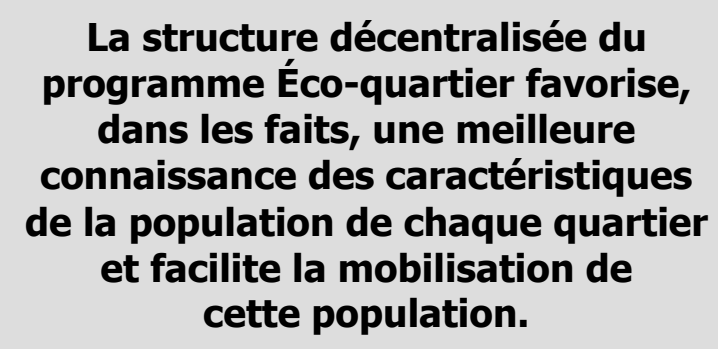

Cependant, la programmation montréalaise est encore peu adaptée aux réalités sociodémographiques des différents quartiers de l'ancienne ville de Montréal. Les organismes Éco-quartiers n'ont actuellement pas les ressources ni la latitude leur permettant d'agir en fonction des besoins et des spécificités de la population qui habite les districts auxquels ils sont associés. Les interventions des Écoquartiers sont déterminées par une convention établie avec l'administration municipale qui s'applique à l'ensemble des quartiers. Par conséquent, elles ne sont pas toujours «calibrées en fonction des réalités locales $\gg{ }^{10}$. Les attentes du programme sont les mêmes envers toutes les composantes de la population, alors que cette population présente des disparités évidentes. En ce sens, la structure décentralisée des Éco-quartiers n'est pas mise à profit pour faci- liter l'adaptation du programme de collecte sélective au milieu local.

\section{Vers une gestion améliorée}

Dans ce contexte, la prise en compte des caractéristiques spécifiques de la population des différents quartiers montréalais par le programme Éco-quartier permettrait de mieux diffuser les informations concernant les modalités et les objectifs de la collecte sélective et elle enverrait aux ménages le message que l'administration municipale comprend les besoins de la population. Une telle reconnaissance pourrait, à son tour, encourager l'adhésion des ménages aux exigences de la collecte sélective et renforcerait l'efficacité de la coproduction du service de gestion des matières recyclables par l'administration municipale et les ménages. De plus, les quartiers où les caractéristiques sociodémographiques sont les plus indicatrices d'une faible intégration culturelle, ceux qui comptent notamment une forte proportion d'individus ne connaissant ni le français ni l'anglais, gagneraient à voir leurs ressources augmenter et les objectifs du programme mieux adaptés à leur contexte. Cette adaptation pourrait permettre aux Écoquartiers de contribuer à une forme d'intégration civique des ménages dans les quartiers ciblés.

Ainsi, certains changements dans la façon de diffuser le message permettraient aux Éco-quartiers de mieux atteindre leur cible. Dans le même sens, la substitution des bacs de recyclage à poignées par des sacs de plastique transparent faciliterait la tâche des ménages. En effet, l'utilisation de sacs de plastique comme contenants de matières recyclables pourrait rendre plus facile le transport des matières récupérées par les ménages, tout en requérant moins d'espace de rangement à l'intérieur des logements. Les sacs de plastique pourraient être particulièrement utiles dans les quartiers à forte densité et où se concentre une proportion élevée de personnes âgées.

Par ailleurs, il semble que les ménages plus nombreux réussissent à réaliser certaines économies d'échelle, faisant en sorte que les quantités de matières recyclables qu'ils produisent sont moins importantes que celles de ménages d'une seule personne. On pourrait envisager de réduire la fréquence 
de la collecte sélective dans les quartiers ou même sur les rues des arrondissements où ces ménages se retrouvent en forte proportion. Une telle diminution de la fréquence de la collecte permettrait aux arrondissements concernés de réduire la facture rattachée à cette activité.

Il serait également possible de prévoir une façon d'approcher les propriétaires d'immeubles à logements multiples afin de les encourager à mieux superviser les activités du tri et à assurer aux locataires les outils dont ils ont besoin pour le faire adéquatement. Ces immeubles sont souvent ceux où la qualité du tri effectué laisse le plus à désirer. Une diminution de la taxe de prélèvement des ordures pour les propriétaires de ces immeubles pourrait ainsi constituer une forme de rétribution et favoriser leur travail actif à ce chapitre.

Dans une optique où elle serait mieux adaptée et renforcée, la formule des Éco-quartiers pourrait s'avérer fort pertinente et elle pourrait être appliquée au sein des autres grandes villes québécoises. En effet, la structure décentralisée du programme montréalais pourrait servir à faciliter la rencontre des objectifs provinciaux de réduction des matières résiduelles (récupérer $65 \%$ des matières résiduelles) dans des villes comme Québec, Gatineau et Saguenay, par exemple.

D'autant plus que certaines mesures ayant permis de renforcer l'efficience et l'efficacité du recyclage dans différentes régions européennes et canadiennes seront dorénavant appliquées au Québec. C'est le cas notamment du partage de la facture de la collecte sélective entre les secteurs privé et public. En France et en Allemagne, par exemple, les gouvernements exigent des industriels qu'ils assument la gestion de la récupération des déchets d'emballages ménagers de même qu'une grande partie de ses coûts. Ainsi, depuis le début des années 1990, les entreprises allemandes qui produisent et distribuent des matériaux d'emballage sont obligées de récupérer et de recycler les emballages qu'elles ont mis en circulation. Une telle obligation a d'ailleurs encouragé ces industriels à se regrouper et à innover dans la mise au point de procédés de tri et de recyclage.
Plus près de chez nous, le gouvernement ontarien a adopté, au mois de juin 2002, un projet de loi prévoyant que les entreprises liées à la production d'emballages versent aux municipalités une compensation financière équivalant à $50 \%$ des coûts des programmes de collecte sélective. Et depuis le mois de décembre 2002, au cours duquel a été adopté le projet de loi 102, c'est au tour du gouvernement québécois d'exiger des entreprises de ce secteur qu'elles assument jusqu'à $50 \%$ des coûts nets de la collecte sélective. Cette nouvelle mesure législative allégera le fardeau des municipalités. Elle représente également un premier pas vers une reconnaissance de la responsabilité des entreprises dans la récupération des matières.

\section{Certains changements dans la façon de diffuser le message permettraient aux Éco-quartiers de mieux atteindre leur cible.}

Ce partage plus équitable de la facture de la collecte a favorisé l'innovation des entreprises dans la recherche de nouvelles pistes en matière de recyclage dans plusieurs pays européens. Conjugué à une bonification des supports offerts aux ménages, le paiement partagé de la facture de la collecte sélective devrait en augmenter l'efficacité au Québec.

\section{Conclusion}

En dépit du fait que le développement durable fasse l'objet d'un consensus de plus en plus large au sein des sociétés occidentales, et malgré qu'il existe de nombreuses expériences réussies en matière de recyclage des résidus domestiques, nous constatons que les habitudes de vie et les comportements sociaux prennent plusieurs années, voire plusieurs décennies à changer. Récemment, lors d'une entrevue avec un journaliste de la presse écrite, le responsable de la ville de Montréal en matière d'environnement soulignait que le recul du recyclage en 1999 s'expliquait par le manque de publicité ${ }^{11}$, ce qui a été corrigé dans les années subséquentes par l'administration municipale. Par la suite, le conseil municipal a également voté un nouveau règlement afin de favoriser le recours au recyclage par les ménages montréalais. 
L'addition de ces deux mesures expliquerait, selon lui, l'augmentation de $36 \%$ du recyclage entre 1999 et 2000 sur l'ensemble du territoire de l'ancienne ville de Montréal.

En passant en revue les activités des Éco-quartiers, nous avons constaté que ces derniers jouent un rôle important dans tous les quartiers urbains en matière de sensibilisation à l'environnement et au recyclage. Pour autant, les ressources mises à la disposition de ces groupes ne sont pas suffisantes pour leur permettre d'être en mesure de tenir compte et de s'adapter aux spécificités tant socioculturelles que sociodémographiques de plusieurs quartiers montréalais. Il en résulte une efficacité plus faible de leur action.

Nous pouvons nous réjouir de l'adoption récente au Québec de mesures dont pouvaient se prévaloir depuis quelques années les administrations locales dans différents pays d'Europe et dans certaines régions canadiennes, tel le partage de la facture de la collecte sélective entre le secteur public et le secteur privé, puisque ces nouvelles dispositions devraient avoir des effets positifs en termes d'amélioration de la collecte sélective à l'échelle locale. En même temps, ces mesures doivent faire partie d'un ensemble intégré. Des approches ciblées en fonction des types de ménages et d'immeubles demeurent nécessaires, comme cela pourrait être envisagé auprès des propriétaires d'immeubles à logements multiples.

\section{Il n'en reste pas moins que l'action des ménages au quotidien autour du recyclage n'est pas une action négligeable, même si elle n'est pas suffisante en soi.}

Depuis que les Éco-quartiers ont été mis sur pied à Montréal au milieu des années 1990, la ville de Montréal a fait l'expérience des possibilités et des limites de la participation des ménages aux activités de tri et de recyclage. Si nous pouvons effectuer un bilan positif de l'action de ces groupes à plus d'un titre, il n'en reste pas moins que leur action pourrait mieux s'adapter aux caractéristiques et aux différences des quartiers urbains montréalais.
En outre, même si le recyclage s'avère un élément important dans la problématique du développement durable, il n'existe pas d'une manière isolée. La collecte sélective qui s'adresse aux ménages des milieux urbains s'inscrit dans un ensemble de pratiques de gestion destinées à traiter les résidus domestiques d'une manière conforme à la problématique du développement durable et à transformer en profondeur les valeurs, les comportements et les pratiques sociales à l'égard de l'environnement. À cet effet, nous devons comprendre que le développement durable défini en termes de justice sociale, d'équilibre écologique et de protection de l'environnement va bien au-delà de la collecte sélective. Il engage des choix de sociétés que ne peuvent faire les ménages à eux seuls. Une responsabilisation des entreprises et des gouvernements supérieurs est également requise. Il n'en reste pas moins que l'action des ménages au quotidien autour du recyclage n'est pas une action négligeable, même si elle n'est pas suffisante en soi.

Pour l'instant, notre intention était de montrer que celle-ci peut s'inscrire dans un plan local de développement durable à condition que les groupes engagés dans sa mise en œuvre disposent des ressources nécessaires à leur action. En ce sens, et en dépit des difficultés qu'ils ont eues à surmonter jusqu'à maintenant, les Éco-quartiers de Montréal constituent un exemple d'innovation en matière d'action collective qu'il nous apparaît utile de mieux connaître et dont pourraient s'inspirer d'autres villes au Québec.

\section{Notes et références}

1 Geneviève Cloutier a fait sa maîtrise en urbanisme à l'Institut d'urbanisme de l'Université de Montréal. Pierre Hamel est professeur au département de sociologie de l'Université de Montréal. Ses recherches en cours portent sur les enjeux métropolitains, les mouvements sociaux et la démocratie locale.

2 Cloutier, G. (2002). L'influence des caractéristiques sociodémographiques et civiques sur l'efficacité de la collecte sélective à Montréal, mémoire de maîtrise, Institut d'urbanisme, Université de Montréal.

3 Rumpala, Y. (1999). « Le réajustement du rôle des populations dans la gestion des déchets ménagers », Revue française de science politique, vol. 49, $\mathrm{n}^{\text {os }} 4-5$, p. 601-630. 
4 Le Programme fait en sorte que les 51 districts électoraux de l'ancienne ville de Montréal sont desservis par un intervenant Éco-quartier. Cependant, en réalité, 37 organismes étaient mandataires du Programme en 2001-2002.

5 Ville de Montréal (2001). Gestion des matières résiduelles. Bilan 2000, Services des travaux publics et de l'environnement, Division de l'environnement.

6 Côté, C. (2003). « Le recyclage en hausse à Montréal », La Presse, mercredi 15 janvier, p. A9.

7 En 2000, les quartiers de Montréal où l'on enregistrait les plus importantes quantités de matières récupérées à la collecte sélective se distinguaient par leurs proportions plus élevées d'individus ayant fait des études universitaires et connaissant le français et l'anglais. Cloutier, G. (2002), op. cit., p. 84.

8 Corriveau, J. (2002). « Montréal est engagé dans une course contre la montre », Le Devoir, mercredi 7 août, p. A1 et A8.

9 Le taux de récupération est de $58 \%$ à Guelph. Site Internet du système « wet-dry » :

http://www.city.guelph.on.ca/pdf/wd_factsht.pdf

10 Sénécal, G. et al. (2001). Évaluation des retombées socioéconomiques du programme Éco-quartier de la Ville de Montréal en 2000, INRS-Urbanisation, Culture et société, p. 36.

11 Côté, C., op. cit., p. A9. 